\title{
A New Base Station Receiver for Increasing Diversity Order in a CDMA Cellular System
}

\author{
Wan Choi, Chaehag Yi, Jin Young Kim, and Dong In Kim, Senior Member, IEEE
}

\begin{abstract}
A new base station receiver is proposed and analyzed for a code-division multiple-access (CDMA) cellular system. The proposed receiver can achieve remarkable diversity gain by increasing diversity order with reasonable cost and complexity. From the numerical results, it is confirmed that the proposed receiver structure can be a practical solution for enhancing reverse-link capacity and improving performance in CDMA cellular system operations. The result in the letter can find its applications to legacy IS-95/cdma2000 1x base stations with simple modifications.
\end{abstract}

Index Terms-Code-division multiple access (CDMA), diversity, multipath fading, receiver complexity.

\section{INTRODUCTION}

$\mathbf{S}$ EVERAL diversity techniques have been studied and found practical use in many communication systems in order to combat multipath fading and improve performance. Among the techniques, spatial diversity has been commonly used at cellular base station (BS) receivers due to its simplicity in implementation [1].

Although it is well known that higher order diversity can improve the receiver performance, the second-order spatial diversity with two receiving antennas at a BS has been most popular, because the higher order spatial diversity at a BS requires additional cost associated with strict zoning requirements. The antenna elements for the spatial diversity need to be separated at least ten times wavelength in order to obtain signals that fade independently [1]. The possibility of using polarization diversity has been studied with a motivation that the polarization diversity using a dual-polarized antenna can reduce cost and space for installation, compared with the traditional spatial diversity. The previous investigations on polarization diversity have revealed that the polarization diversity is able to achieve comparable performance to the spatial diversity [2]-[5].

Exploring the polarization as an additional source of diversity has been considered in [6] and [7]. Particularly, [7] investigated the benefit of increased diversity order using spatially separated polarized antennas at the $\mathrm{BS}$ receiver of a code-division multipleaccess (CDMA) cellular system. The paper proved that the four-

Paper approved by V. A. Aalo, the Editor for Diversity and Fading Channel Theory of the IEEE Communications Society. Manuscript received December 30, 2002; revised June 9, 2003 and February 16, 2004.

W. Choi was with KT Freetel, Inc., Seoul, Korea. He is now with the Department of Electrical Engineering, The University of Texas at Austin, Austin, TX 78712 USA (e-mail: wchoi@mail.utexas.edu).

C. Yi is with Solid Technologies, Inc., Seoul 138-803, Korea (e-mail: steveyi@st.co.kr).

J. Kim is with Kwangwoon University, Seoul 139-701, Korea (e-mail: jinyoung@daisy.ac.kr).

D. Kim is with the School of Engineering Science, Simon Fraser University, Burnaby, BC V5A 1S6, Canada (e-mail: dikim@sfu.ca).

Digital Object Identifier 10.1109/TCOMM.2004.836595 branch diversity receiver combining spatial and polarization diversity could considerably improve receiver performance and enhance reverse-link capacity. However, implementation of the four-branch diversity receiver using spatially separated cross-polarized antennas requires high cost and considerable modification, compared with the conventional dual-diversity receiver. The four-branch diversity receiver also needs additional hardware units and space for increased diversity branches.

In this letter, we propose a new BS receiver to increase diversity order with low cost and complexity, and analyze the performance of the proposed receiver. The bit-error probability (BEP) and the outage probability are derived and compared for each type of receivers.

The letter is organized as follows. In Section II, the proposed new BS receiver is shown and described. In Section III, outage probability and BEP are derived for the proposed receiver. In Section IV, some numerical results are presented. The conclusions are drawn in Section V.

\section{Proposed Receiver Structure}

In sectorized cellular CDMA systems, a typical BS supports three sectors, and the structure for the four-branch diversity receiver using spatially separated dual-polarized antennas in [7] is shown in Fig. 1(a). In each sector, the polarized antennas form four diversity branches and the signals from each branch are down-converted and digitized in the radio frequency (RF) and the intermediate frequency (IF) circuits. Then, they are transfered to the baseband RAKE receiver for matched filtering and combining.

The four-branch diversity receiver requires additonal hardware units for the increased diversity branches, compared with the conventional dual-diversity receiver, because the signals from antenna elements have the same pseudonoise (PN) code offsets and suffer independent fading.

In cellular CDMA systems, though the transmitted signal from a mobile station (MS) is spread by a user-specific PN code, the received signals from the MS have different PN offsets due to the multipath components. The RAKE receiver in the baseband modem resolves the multipath signals and combines them [8]. Even if the signals with the different PN offsets are combined, the RAKE receiver can resolve the combined signals based on the PN code offsets. With this motivation, we propose a new BS receiver that can increase diversity order with low cost and complexity. The structure of the proposed receiver is shown in Fig. 1(b). The RF signal from one antenna element of a cross-polarized antenna is intentionally delayed by the amount of a predetermined value and added by the RF signal from the other antenna element. In the proposed receiver, $M / 2$ branches are for normal branches, and the remaining $M / 2$ 


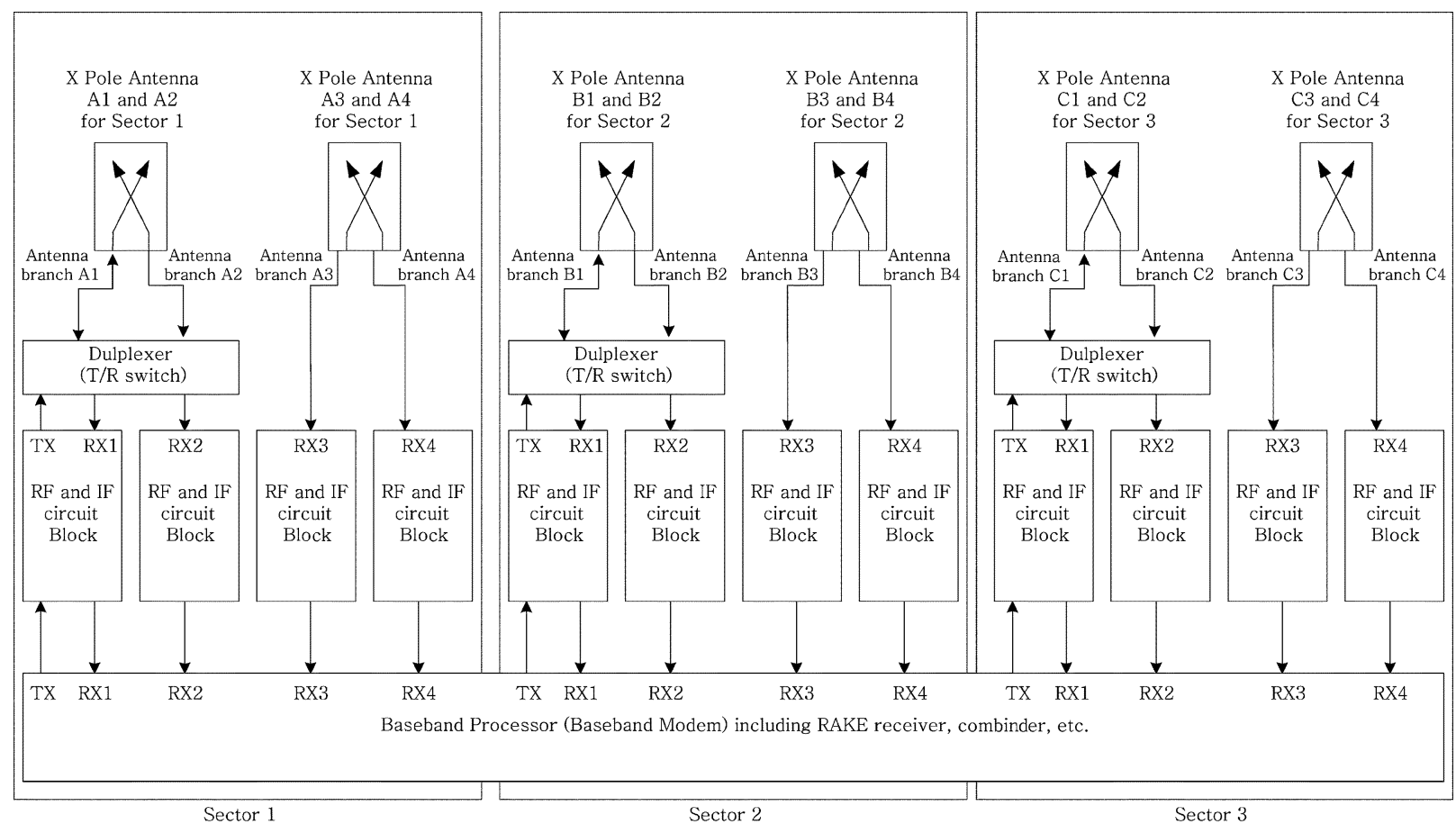

(a) 4-branch diversity receiver

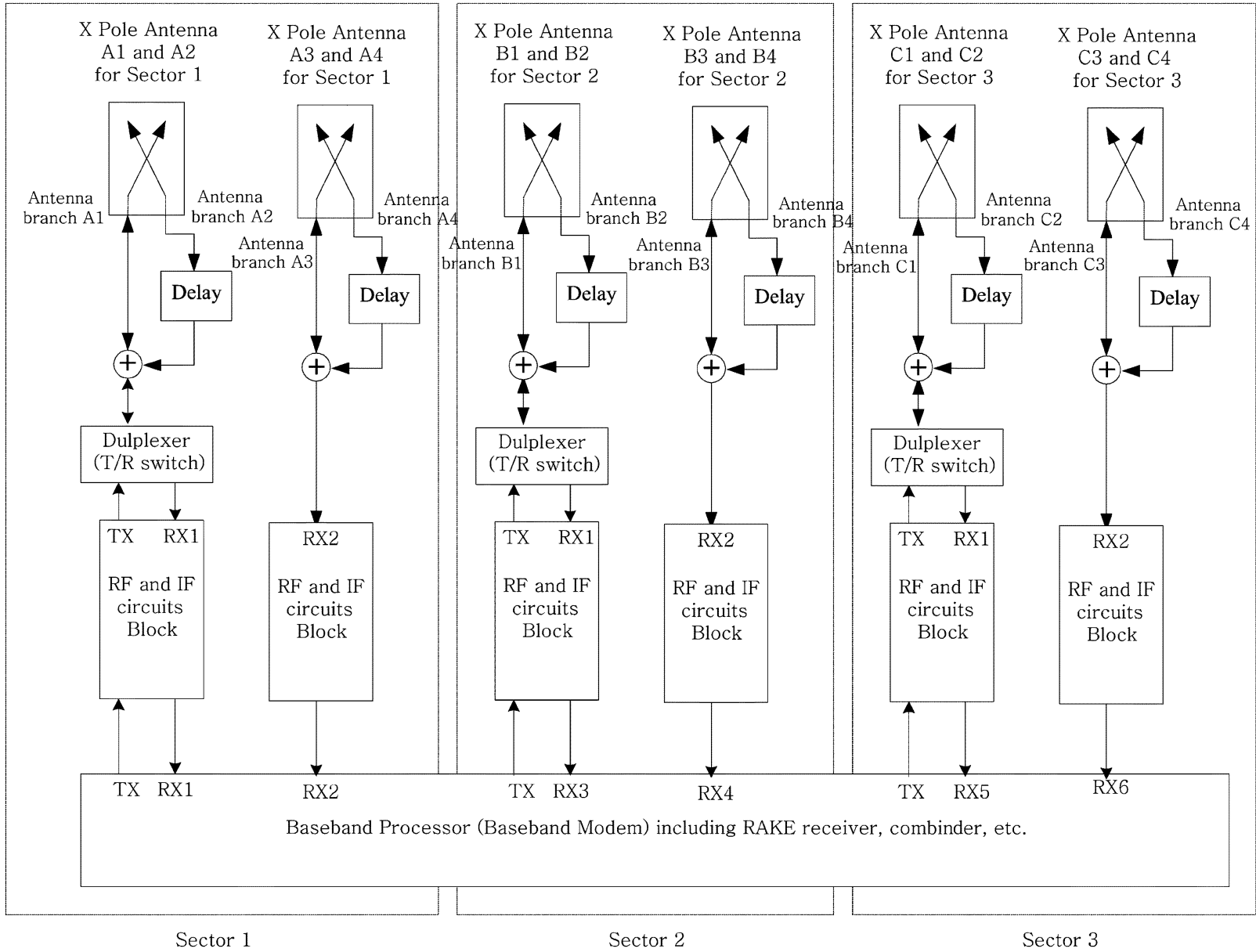

(b) Proposed diversity receiver

Fig. 1. BS receiver structures. (a) Four-branch diversity receiver. (b) Proposed diversity receiver.

branches are for the delay branches among the total $M$-diversity branches $[M=4$ for Fig. 1(b)]. Then, the combined signal in the RF level is input to the RF and IF units for down-conversion and digitization, and transferred to the baseband $\mathrm{RAKE}$ receiver. 
All of the signals from $M$ diversity branches can be resolved in the RAKE receiver, because the sufficient delay can make the fading statistics appear independent even for the same user, and assure the resolvability as well. The predetermined delay should be larger than the maximum excess path delay for the meaningful multipath signals from the normal branches, in order to avoid overlapping with the intentionally delayed signals from the delay branches. The intentional RF delay module can be deployed with either active components or passive components.

The proposed receiver can be easily applied to the legacy BSs with some modification in the antenna part, and does not need additional hardware units and space, compared with the fourbranch diversity receiver in Fig. 1(a). The additional hardware units and the space for increasing the number of branches cost too much burden in implementing the BS.

\section{Performance ANALysis}

In this section, the outage probability and BEP are derived for each type of receiver by modifying the results of [9] and [10]. Although the synchronous assumption is not valid in commercial CDMA systems, a synchronous reverse link is assumed in the performance analysis because it allows a complete comparative analysis of the diversity techniques. In addition, the following assumptions are made for simplicity of analysis: 1) all the signals from antenna elements are independent; 2) power control and channel parameters estimated for maximum ratio combining (MRC) are perfect; 3) the multipath fading channel is modeled as single-path Rayleigh fading; and 4) the crosscorrelation factor between different user-specific PN codes is $\rho$. In the synchronous reverse link, the user can be effectively distinguished by appropriately cyclic shifted sequences of a PN sequence, and the crosscorrelation factor $\rho$ between user-specific codes can be equivalent to the autocorrelation factor.

\section{A. Conventional M-Branch Diversity Receiver}

For the $M$-branch diversity receiver, matched-filter (MF) output for a user 0 can be given in a vector form by

$$
\mathbf{Z}_{0}(n)=s_{0}(n) \mathbf{A}_{0}+\sum_{i=1}^{N} s_{i}(n) \mathbf{A}_{i}+\mathbf{n}(n)
$$

where each element of $\mathbf{Z}_{0}(n)$ represents the MF output corresponding to each diversity branch. $s_{i}(n)(i=0,1, \ldots, N)$ is a transmitted data sequence of user $i, \mathbf{A}_{1}(i=0,1, \ldots, N)$ is an $M$-dimensional vector which represents filtered outputs of the attenuated signals for the user $i, N$ is the number of interfering users, and $\mathbf{n}(n)$ is an $M$-dimensional Gaussian zeromean vector with covariance matrix $\sigma^{2} \mathbf{I}$. Each component of vector $\mathbf{A}_{i}$ is assumed to follow a complex Gaussian distribution with mean power of $P_{i}$. For a perfect power-controlled system, $P_{0}=P, P_{i}=\rho P \forall i(i \neq 0)$.

In an MRC system, the signal of each branch is weighted by a corresponding complex-valued channel gain before combining.
Thus, the weighting vector for the MF output given in (1) becomes $\mathbf{A}_{0}$. Then, the output signal-to-interference-plus-noise ratio of the MRC system (SINR_o) is given by

$$
\gamma=\frac{\mathbf{A}_{0}^{H} \mathbf{A}_{0} \mathbf{A}_{0}^{H} \mathbf{A}_{0}}{\mathbf{A}_{0}^{H} \mathbf{C}_{n n} \mathbf{A}_{0}}=\frac{x_{0}}{\sum_{i=1}^{N} z_{i}+\sigma^{2}}
$$

where the superscript ${ }^{H}$ denotes complex conjugate transpose, and $\mathbf{C}_{n n}=\sum_{i=1}^{N} \mathbf{A}_{i} \mathbf{A}_{i}^{H}+\sigma^{2} \mathbf{I}, x_{0}=\mathbf{A}_{o}^{H} \mathbf{A}_{0}$, and $z_{i}=$ $\left|\mathbf{A}_{o}^{H} \mathbf{A}_{i}\right|^{2} / x_{0}$. The SINR_o in (2) has a similar form to that of [9] and [10]. From the results of [9] and [10], the probability density function (pdf) of the SINR_o can be easily obtained as

$$
f_{\gamma}(\gamma)=\int_{0}^{\infty}\left(z+\sigma^{2}\right) f_{X_{0}}\left(\left(z+\sigma^{2}\right) \gamma\right) f_{Z}(z) d z
$$

where $f_{x_{0}}\left(x_{0}\right)$ and $f_{z}(z)$ are the pdfs of $x_{0}$ and $z=\sum_{i=1}^{N} z_{i}$, respectively, and are given by

$$
\begin{aligned}
f_{X_{0}}\left(x_{0}\right) & =\frac{1}{P_{0}^{M} \Gamma(M)} x_{0}^{M-1} e^{\left(\frac{-x_{0}}{P_{0}}\right)} \\
f_{Z}(z) & =\frac{z^{N-1}}{\Gamma(N)(\rho P)^{N}} e^{-\frac{z}{\rho P}} .
\end{aligned}
$$

The outage probability is the probability that the received signal level falls below a specified level $\beta$, at which the desired service quality cannot be satisfied. From (3), the outage probability $P_{o}$ is obtained by [10]

$$
\begin{aligned}
P_{o}(N)= & \int_{0}^{\beta} f_{\gamma}(\gamma) d \gamma \\
= & \int_{0}^{\infty} \int_{0}^{\left(w+\sigma^{2}\right) \beta} f_{X_{0}}(\gamma) f_{Z}(w) d \gamma d w \\
= & 1-\left(\frac{\rho^{-1}}{\beta+\rho^{-1}}\right)^{N} \exp \left(-\frac{\beta}{\Omega_{0}}\right) \sum_{k=0}^{M-1} \sum_{i=0}^{k}\left(\begin{array}{c}
k \\
i
\end{array}\right) \\
& \times \frac{\Gamma(N+i)\left(\frac{\beta}{\Omega_{0}}\right)^{k}}{\Gamma(N) k !}\left(\frac{\Omega_{0}}{\beta+\rho^{-1}}\right)^{i}
\end{aligned}
$$

where $\Omega_{0}=P / \sigma^{2}$ and $P_{o}(N)$ denotes explicitly the dependence of $P_{o}$ on $N$.

For the MRC, the BEP can be obtained by GA for multipleaccess interference (MAI). In (1), the second term represents the MAI and can be approximated as a Gaussian random variable from the result of [11]. Then, the MAI plus additive white Gaussian noise (AWGN) is also a Gaussian random variable, and the BEP can be given by [12]

$$
P_{e}(\bar{\gamma})=\left[\frac{1}{2}(1-\mu)\right]^{M} \sum_{k=0}^{M-1}\left(\begin{array}{c}
M-1+k \\
k
\end{array}\right)\left[\frac{1}{2}(1+\mu)\right]^{k}
$$

where $\mu=\sqrt{\bar{\gamma} /(1+\bar{\gamma})}$ and $\bar{\gamma}$ is an average SINR per branch (SINR_br). 


\section{B. Proposed M-Branch Diversity Receiver}

For the proposed receiver, the RAKE receiver resolves the signals of each diversity branch from the coupled signal. The MF output for a user 0 can be divided into two parts, which are the resolved MF outputs for the signals from normal and delay branches, respectively, and given by

$$
\mathbf{Z}_{0}(n)=\left[\begin{array}{l}
\mathbf{Z}_{0}^{(1)}(n) \\
\mathbf{Z}_{0}^{(2)}(n)
\end{array}\right]
$$

where $\mathbf{Z}_{0}^{(1)}$ and $\mathbf{Z}_{0}^{(2)}$ are $M / 2$-dimensional vectors, and can, respectively, be given by

$$
\begin{aligned}
\mathbf{Z}_{0}^{(1)}(n)= & s_{0}(n) \mathbf{A}_{0}^{(1)}+s_{0}\left(n-\left\lfloor\frac{\delta}{T_{s}}\right\rfloor\right) \rho \mathbf{A}_{0, \delta}^{(2)} \\
& +\sum_{i=1}^{N} s_{i}(n) \mathbf{A}_{i}^{(1)} \\
& +\sum_{i=1}^{N} s_{i}\left(n-\left\lfloor\frac{\delta}{T_{s}}\right\rfloor\right) \mathbf{A}_{i, \delta}^{(2)}+\mathbf{n}^{(1)}(n) \\
\mathbf{Z}_{0}^{(2)}(n)= & s_{0}\left(n+\left\lfloor\frac{\delta}{T_{s}}\right\rfloor\right) \rho \mathbf{A}_{0, \delta}^{(1)}+s_{0}(n) \mathbf{A}_{0}^{(2)} \\
& +\sum_{i=1}^{N} s_{i}\left(n+\left\lfloor\frac{\delta}{T_{s}}\right\rfloor\right) \mathbf{A}_{i, \delta}^{(1)} \\
& +\sum_{i=1}^{N} s_{i}(n) \mathbf{A}_{i}^{(2)}+\mathbf{n}^{(2)}(n)
\end{aligned}
$$

where each element $\mathbf{Z}_{0}^{(1)}$ of $\mathbf{Z}_{0}^{(2)}$ represents the MF output corresponding to normal and delay diversity branches, respectively. In the equations, the $\delta$ denotes the intentional delay, $T_{s}$ denotes a sampling time, and $\lfloor\cdot\rfloor$ denotes the floor function. $\mathbf{A}_{i}^{(1)}(i=$ $0,1, \ldots, N)$ and $\mathbf{A}_{i}^{(2)}(i=0,1, \ldots, N)$ are $M / 2$-dimensional vectors which represent filtered outputs of the attenuated signal of user $i$ from the normal and delay branches, respectively. The $\mathbf{n}^{(1)}(n)$ and $\mathbf{n}^{(2)}(n)$ are $M / 2$-dimensional Gaussian vectors with zero mean and covariance matrix $\sigma^{2} \mathbf{I}$. Each component of vector $\mathbf{A}_{i}^{(1)}$ and $\mathbf{A}_{i}^{(2)}$ is assumed to follow a complex Gaussian distribution with mean power of $P_{i}(=\rho P)$. The relationship between the intentional delay $\delta$ and the crosscorrelation factor $(=\rho)$ is given by

$$
\rho= \begin{cases}=-\frac{1}{L}, & |\delta| \geq T_{c} \\ =-\left(1+\frac{1}{L}\right) \delta+1, & |\delta|<T_{c}\end{cases}
$$

where $L$ and $T_{c}$ denote the length of user-specific PN codes and the chip duration, respectively. The crosscorrelation factor $\rho$ is minimized for $|\delta| \geq T_{c}$, and thus, intentional delay should be larger than $T_{c}$ but smaller than the shifted value for user distinction. The proposed scheme may insert sufficient delay to make the fading statistics appear independent even for the same user, and assure the resolvability as well.

In the matched filtering for user 0's signals from the normal branches, user 0's signals from the delay branches act as another MAI source given in the fourth term in (9). Because the self-interference in the second term in (9) is statistically equivalent to the crosscorrelated interference between different users, the crosscorrelation appears stationary, and the signals from the diversity branches are independent, (9) and (10) can be effectively approximated to

$$
\begin{aligned}
& \mathbf{Z}_{0}^{(1)}(n) \cong s_{0}(n) \mathbf{A}_{0}^{(1)}+\sum_{i=1}^{2 N+1} s_{i}(n) \mathbf{A}_{i}^{(1)}+\mathbf{n}^{(1)}(n) \\
& \mathbf{Z}_{0}^{(2)}(n) \cong s_{0}(n) \mathbf{A}_{0}^{(2)}+\sum_{i=1}^{2 N+1} s_{i}(n) \mathbf{A}_{i}^{(2)}+\mathbf{n}^{(2)}(n) .
\end{aligned}
$$

From (12) and (13), the MF output for a user 0 can be given in an $M$-dimensional vector, and given by

$$
\mathbf{Z}_{0}(n)=s_{0}(n) \mathbf{A}_{0}+\sum_{i=1}^{2 N+1} s_{i}(n) \mathbf{A}_{i}+\mathbf{n}(n)
$$

where

$$
\mathbf{Z}_{0}(n)=\left[\begin{array}{l}
\mathbf{Z}_{0}^{(1)}(n) \\
\mathbf{Z}_{0}^{(2)}(n)
\end{array}\right], \mathbf{A}_{i}(n)=\left[\begin{array}{c}
\mathbf{A}_{i}^{(1)}(n) \\
\mathbf{A}_{i}^{(2)}(n)
\end{array}\right], \mathbf{n}(n)=\left[\begin{array}{l}
\mathbf{n}^{(1)}(n) \\
\mathbf{n}^{(2)}(n)
\end{array}\right] .
$$

The resolved MF output in (14) is similar to that of the conventional diversity receiver, except that the MAI increases by a factor of $(2+1 / N)$. For the MRC, the weighting vector in (14) becomes $\mathbf{A}_{0}$, and the SINR_o is given by (2) with $N$ replaced by $(2 N+1)$. Therefore, the outage probability is obtained by $P_{o}(2 N+1)$ in $(6)$.

In (14), the second term represents the MAI and can be approximated as a Gaussian random variable. When the average SINR_br of the conventional receiver is $\bar{\gamma}\left(=P /\left(N \rho P+\sigma^{2}\right)\right)$, the average SINR_br for the proposed receiver is given by

$$
\begin{aligned}
\bar{\gamma}_{\text {new }} & =\frac{P}{(2 N+1) \rho P+\sigma^{2}} \\
& =\frac{1}{\frac{\left(2+\frac{1}{N}\right)}{\bar{\gamma}}-\frac{\left(1+\frac{1}{N}\right) \sigma^{2}}{P}} \\
& =\frac{1}{\frac{\left(2+\frac{1}{N}\right)}{\bar{\gamma}}-\frac{\left(1+\frac{1}{N}\right)}{\Omega_{0}}} .
\end{aligned}
$$

Then the BEP of the proposed receiver can be obtained as $P_{e}\left[\bar{\gamma}_{\text {new }}\right]$ in (7). For the case where the MAI becomes dominant, the average SINR_br becomes $\bar{\gamma} /(2+1 / N)$ for the proposed receiver, and the BEP of the proposed receiver can be obtained as $P_{e}[\bar{\gamma} /(2+1 / N)]$ in $(7)$.

\section{On the Validity of Gaussian Approximation}

Though we use the Gaussian approximation (GA) in deriving the BEP, the GA can be inaccurate when the transmitted date sequence is random, unless the number of interfering users is large. In the situations where the GA is not appropriate, a more in-depth analysis considering conditional BEP on the interference terms must be applied. But it may be very difficult to obtain a closed-form result by this approach. The method to improve the inaccuracy of GA in this situation remains our further study. However, considering the fact that the purpose of this letter is to investigate the feasibility of the proposed receiver, the derived BEP with the GA can be useful in comparative analysis by itself. For the conventional diversity receiver, the BEP with the GA can be regarded as only an optimistic loose bound because of the inaccuracy of the GA. But for the proposed receiver, the inaccuracy of the GA is obviously improved, since the proposed 


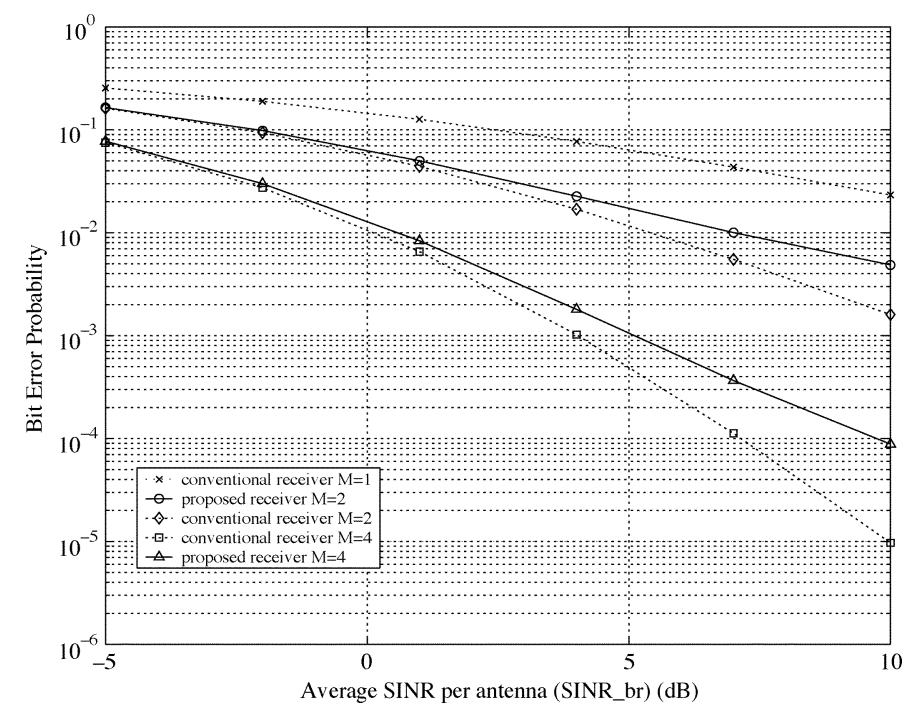

Fig. 2. BEP versus average SINR_br.

receiver causes the effect of increasing the number of interfering users $(N \rightarrow 2 N+1)$.

\section{NUMERICAL RESULTS}

Some numerical results for the BEP and outage probability are presented and compared for the conventional and the proposed diversity receivers. To show the feasibility of the latter, we are more concerned with the comparison between conventional two-branch diversity and proposed diversity receivers.

Fig. 2 shows the BEP versus average SINR_br in a Rayleigh fading channel environment in the general case, including the situation where the MAI is not dominant compared with AWGN. For a BEP of $10^{-3}$, the four-branch receiver has about $1-\mathrm{dB}$ gain over the proposed four-branch receiver, while the proposed four-branch receiver has about 5-dB gain over the conventional two-branch receiver. Though the four-branch receiver shows better performance than any other receivers considered, it requires high cost and considerable complexity, compared with the conventional two-branch receiver. Meanwhile, the proposed four-branch receiver can achieve a reasonable BEP gain over the conventional two-branch receiver with low cost and complexity. The performance gain becomes larger as the average SINR_br increases. This gain can directly result in capacity and coverage increases in the mobile cellular system operations.

Fig. 3 shows the outage probability versus SNR $\left(\Omega_{0}=\right.$ $\left.P_{0} / \sigma^{2}\right)$ as another performance measure. Five interfering users, 5-dB outage threshold SINR, and 128-length user-specific codes are assumed for all the types of receivers for performance comparison. For an outage probability of $10^{-2}$, the gap of the required SNR between the proposed four-branch receiver and the conventional two-branch receiver is about $15 \mathrm{~dB}$. That means the received power level of the conventional two-branch receiver should be $15 \mathrm{~dB}$ larger than that of the proposed four-branch receiver at the same level of AWGN to achieve the same outage probability of $10^{-2}$. Thus, transmit power of the MS in the proposed four-branch receiver can be $15 \mathrm{~dB}$ less than that of the conventional two-branch receiver.

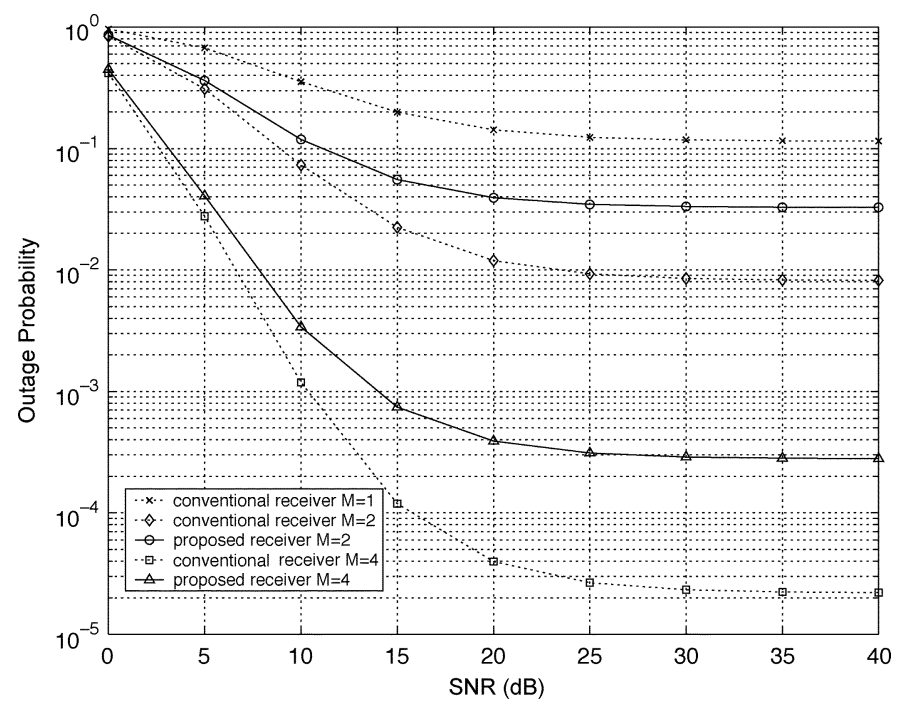

Fig. 3. Outage probability versus SNR.

The gap may depend on average outage threshold SINR_o $(\beta)$, number of interfering users, and target outage probability. The saved power can extend the lifetime of the battery in the MS and reduce the interfering power to other users. Although MAI is not always dominant compared with AWGN in the synchronous reverse link, MAI becomes dominant, and the average SINR_o converges on a certain level as the SNR increases. In the predetermined average outage threshold SINR_o condition, the converged SINR_o also makes the outage probability converge on a certain probability. The converged value of outage probability will depend on the value of the predetermined average outage threshold SINR_o. From the figure, the outage probability versus SINR can be easily derived, using the relationship between the average SINR_o and SNR, $\bar{\gamma}=\Omega_{0} /\left(\sum_{i=1}^{N} \Omega_{i}+1\right)$, where $\Omega_{i}=P_{i} / \sigma^{2}=\rho \bar{P} / \sigma^{2}$.

From the BEP and outage performance shown in Figs. 2 and 3 , the proposed four-branch diversity receiver can achieve remarkable gains over the conventional two-branch diversity receiver with low cost and complexity, and the gains can directly be linked to a capacity enhancement.

\section{CONCLUSION}

In this letter, the new BS receiver has been proposed and analyzed in order to increase diversity order in the CDMA cellular system. The BEP and outage probability are evaluated and compared for the conventional and the proposed diversity receivers. From the numerical results, it has been demonstrated that the proposed receiver can achieve remarkable diversity gain with reasonable cost and complexity. The proposed receiver is expected to provide a practical solution for enhancing reverselink capacity and improving performance in the CDMA cellular system operations. The proposed receiver can be applied to the legacy IS-95/cdma2000 1x BSs with simple modifications.

\section{ACKNOWLEDGMENT}

The authors would like to thank R. Padovani of Qualcomm Inc. for his helpful discussion and encouraging appreciation of 
our work. They also appreciate the comments of the editor and reviewers.

\section{REFERENCES}

[1] W. C. Jakes, Microwave Mobile Communications. New York: Wiley, 1974.

[2] W. C. Y. Lee and Y. S. Yeh, "Polarization diversity system for mobile radio," IEEE Trans. Commun., vol. COM-26, pp. 912-923, Oct. 1972.

[3] R. G. Vaughan, "Polarization diversity in mobile communications," IEEE Trans. Veh. Technol., vol. 39, pp. 177-186, Aug. 1990.

[4] A. M. D. Turkmani, A. A. Arowojolu, P. A. Jefford, and C. J. Kellen, "An experimental evaluation of the performance of two-branch space and polarization diversity scheme at $1800 \mathrm{MHz}$," IEEE Trans. Veh. Technol., vol. 44, pp. 318-326, May 1995.

[5] B. Lindmark and M. Nilsson, "On the available diversity gain from different dual-polarized antennas," IEEE J. Select. Areas Commun., vol. 19, pp. 287-294, Feb. 2001.
[6] G. I. Siquiera et al., "Combined use of space and polarization diversity on mobile cellular network," in Proc. IEEE GLOBECOM, Rio de Janeiro, Brazil, Dec. 1999, pp. 863-867.

[7] L. Aydin, E. Esteves, and R. Padovani, "Reverse link capacity and coverage improvement for CDMA cellular systems using polarization and spatial diversity," in Proc. IEEE Int. Conf. Communications, New York, NY, Apr. 2002, pp. 1887-1892.

[8] G. L. Turin, "Introduction to spread spectrum antimultipath techniques and their application to urban digital radio," Proc. IEEE, vol. 68, pp. 328-353, Mar. 1980.

[9] J. Cui and A. U. H. Sheikh, "Outage probability of cellular radio systems using maximal ratio combining in the presence of multiple interferers," IEEE Trans. Commun., vol. 47, pp. 1783-1787, Aug. 1999.

[10] V. A. Aalo and C. Chayawan, "Outage probability of cellular radio systems using maximal ratio combining in Rayleigh fading channel with multiple interferers," IEE Electron. Lett., vol. 36, pp. 1314-1315, July 2000.

[11] J. S. Lehnert and M. B. Pursley, "Error probabilities for binary direct sequence spread-spectrum communication with random signature sequences," IEEE Trans. Commun., vol. COM-35, pp. 87-98, Jan. 1987.

[12] J. G. Proakis, Digital Communications, 4th ed. New York: McGrawHill, 2001. 